\title{
The microbiome's relationship with congenital heart disease: more than a gut feeling
}

\author{
Dan Feng ${ }^{1}$, Jason T. Christensen ${ }^{1}$, Anji T. Yetman ${ }^{1,2}$, Merry L. Lindsey ${ }^{3,4}$, Amar B. Singh ${ }^{4,5}$ and \\ Jeffrey D. Salomon ${ }^{1,3,6^{*}}$
}

\begin{abstract}
Patients with congenital heart disease (CHD) are at risk for developing intestinal dysbiosis and intestinal epithelial barrier dysfunction due to abnormal gut perfusion or hypoxemia in the context of low cardiac output or cyanosis. Intestinal dysbiosis may contribute to systemic inflammation thereby worsening clinical outcomes in this patient population. Despite significant advances in the management and survival of patients with CHD, morbidity remains significant and questions have arisen as to the role of the microbiome in the inflammatory process. Intestinal dysbiosis and barrier dysfunction experienced in this patient population are increasingly implicated in critical illness. This review highlights possible CHD-microbiome interactions, illustrates underlying signaling mechanisms, and discusses future directions and therapeutic translation of the basic research.
\end{abstract}

Keywords: Congenital Heart Disease, Microbiome, Intestinal barrier dysfunction, Systemic inflammation, Cardiopulmonary bypass

\section{Introduction}

Congenital heart disease (CHD) remains an important risk for morbidity and mortality in the pediatric population, accounting for up to $50 \%$ of mortality due to birth defects[1]. Surgical techniques and post-operative management have improved survival to adulthood from 30 to $85 \%$ over the past 30 years, but CHD remains a significant cause of death in patients less than one year of age [2-4]. Contributors to poor outcomes include delayed repair, multiple organ dysfunction from heart failure, infection, prematurity, and arrhythmia [4-7]. Although survival to adulthood continues to improve [8-12], the inflammatory response and low cardiac output syndrome often seen in

\footnotetext{
* Correspondence: jeffrey.salomon@unmc.edu

'Department of Pediatrics, University of Nebraska Medical Center, Omaha USA

${ }^{3}$ Department of Cellular and Integrative Physiology, Center for Heart and Vascular Research, University of Nebraska Medical Center, Omaha, USA Full list of author information is available at the end of the article
}

these patients remain important mediators of disease [13, 14]. Pre- and post-operative inflammatory responses can lead to abnormalities in the intestinal microbiome and contribute to worse outcomes following cardiac surgery.

The intestinal microbiome is important in regulating health and homeostasis [15-18], and its dysregulation (termed intestinal dysbiosis) has been well studied in critical illness [19-21] and the cardiac surgical population [22-24]. With dysbiosis, an imbalance in the normal microflora of the gut occurs. This imbalance has been implicated in autoimmune disorders, inflammatory bowel disease, and obesity [18, 19, 25-27]. Recently, hypertension, stroke, myocardial infarction, diabetes-induced cardiac dysfunction, and heart failure have also been linked to intestinal dysbiosis [28-30].

While interactions between the microbiome and cardiovascular disease continue to grow, $\mathrm{CHD}$ has received little attention in this regard. CHD patients remain at risk for developing intestinal dysbiosis and intestinal 
epithelial barrier dysfunction (EBD) due to numerous stressors including abnormal gut perfusion, hypoxemia, impaired nutrition, and poor cardiac output secondary to abnormal cardiac function, valvar regurgitation, and/ or residual cardiac shunts [31]. Dysbiosis has been shown to be present in patients with CHD [32-34]. It is unclear if dysbiosis or its resolution induces or improves EBD because the effects of probiotics and synbiotics in CHD patients have not been well studied [35, 36]. The interaction between CHD and the microbiome will be discussed throughout this review and is illustrated in Fig. 1.

In this review we examine the composition of the intestinal microbiome, discuss the evidence of the microbiota's relationship with $\mathrm{CHD}$, and discuss future directions for research and therapeutic interventions to improve outcomes.

\section{The Microbiome}

The microbiome is a complex system interacting with every organ system in the human body [15]. The intestinal microbiome is populated by $\sim 100$ trillion bacteria from greater than 2000 species. These bacteria co-exist and form an integral aspect of homeostasis [37]. There are six main bacterial phyla which exist in the intestinal tract: Bacteroidetes, Firmicutes, Actinobacteria, Proteobacteria, Fusobacteria, and Verrucomicrobia. In a healthy individual, Bacteroidetes and Firmicutes make up roughly $90 \%$ of the bacterial composition [38]. Proteobacteria, a group of pro-inflammatory opportunistic and

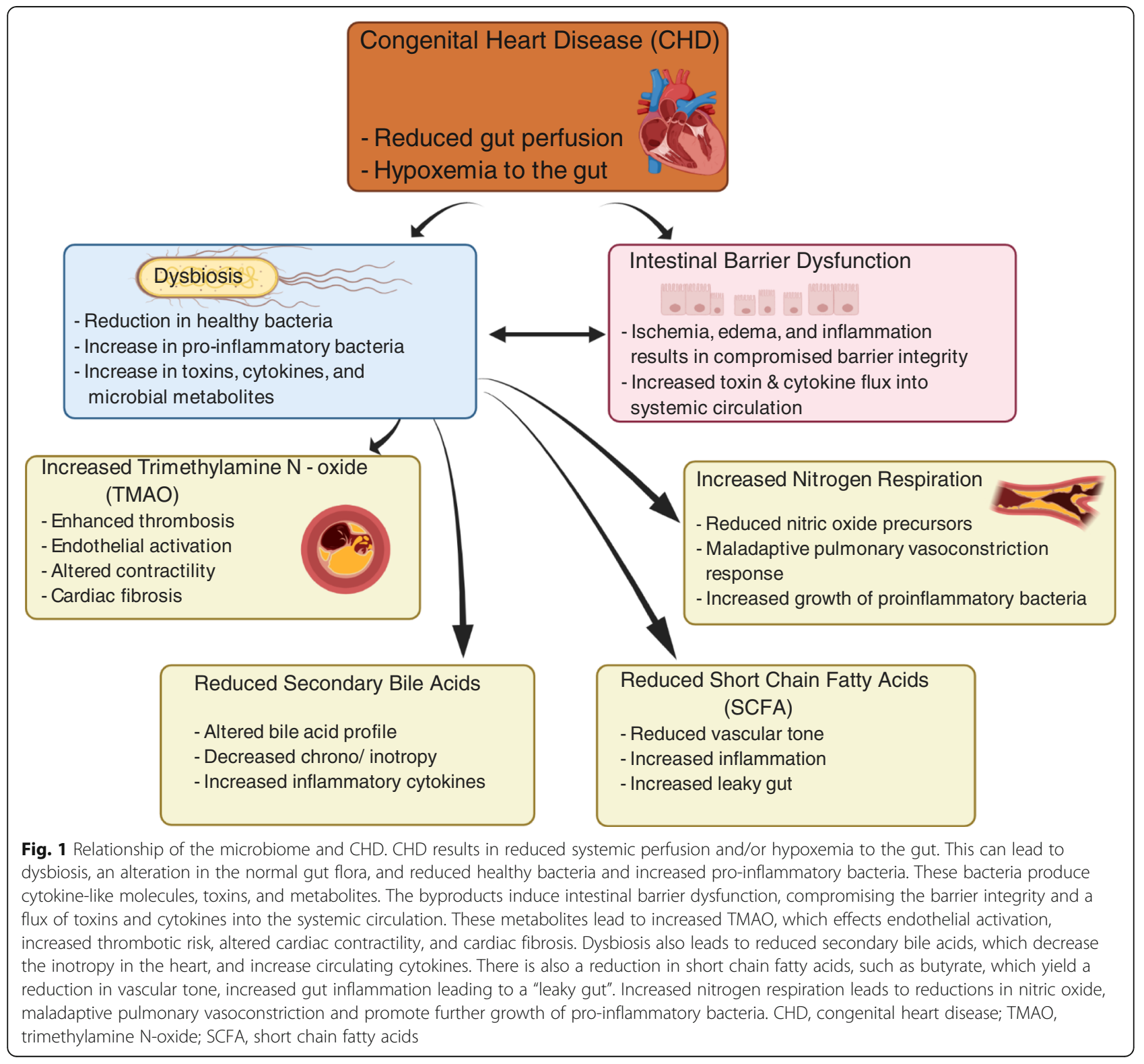


pathogenic bacteria, makes up less than $5 \%$ of the bacterial population. Proteobacteria include organisms associated with disease such as E. coli, Shigella, Salmonella, Klebsiella, and Pseudomonas [39].

Bacteroidetes and Firmicutes, the predominant constituents, are integral to maintaining gut health. A crude measure of microbiome well-being is the Firmicutes to Bacteroidetes $(\mathrm{F} / \mathrm{B})$ ratio. In infants, the $\mathrm{F} / \mathrm{B}$ ratio is $\sim 0.4$, while in adults, it is $\sim 10$ indicating that Firmicutes contribution increases with maturity [40]. Higher F/B ratios have been associated with obesity, coronary artery disease, stroke, heart failure, and autoimmune disease $[26,41]$.

Organisms within different bacterial phyla regulate gut health through a variety of mechanisms. Increases in pro-inflammatory bacteria are associated with a decrease in bile acid synthesis and correlate with EBD [42, 43]. Beneficial bacteria, such as Lactobacillus reuteri and Lactobacillus rhamnosus, assist in developing the intestinal mucus barrier, an important defense system for the gut [43]. Similarly, small molecule signaling between bacterial organisms and host cells via butyrate, trimethylamine $\mathrm{N}$-oxide (TMAO), nitric oxide, cytokines, and endotoxins have been reported. The magnitudes of these signals are dynamic in cardiovascular diseases.

\section{The Microbiome and Cardiovascular Disease}

The gut microbiome is implicated in a variety of cardiovascular disease states, from hypertension and stroke to heart failure [44]. Elevations of pro-inflammatory bacteria, Proteobacteria, in the blood have been associated with an increased risk of developing cardiovascular diseases [45]. Dysbiosis is a noted factor in the development of atherosclerosis through increases in endotoxins and cytokines. Lipopolysaccharides (LPS) and peptidoglycans activate reactive oxygen species and inflammatory pathways when they are released into the bloodstream [46]. These reactive oxygen species can induce arrhythmias, cardiac remodeling through apoptosis and necrosis, endothelial smooth muscle hypertrophy, and oxidative damage to endothelial cells $[47,48]$. Increased amounts of Enterobacteriaceae, a large family of pathogenic and LPS-producing organisms within the phylum Proteobacteria, concurrently with reduced amounts of Bacteroidetes have been noted in patients with identified atherosclerotic cardiovascular disease [49, 50]. Patients with heart failure typically have intestinal wall edema and reduced gut perfusion. This can lead to bacterial translocation through deregulated gut barrier, cytokine production, and endotoxin absorption, which have also been linked to progressive heart failure [51]. This positive feedback loop of heart failure inducing dysbiosis and dysbiosis exacerbating heart failure is an important mechanism of disease severity. All of these changes to the intestinal microbiome can influence the development and exacerbation of multiple cardiovascular disease states.

Other signaling molecules produced by gut microbiota (e.g. TMAO, butyrate, NO) also have cardiovascular effects. Trimethylamine (TMA) is produced by intestinal microbiota in the Firmicutes and Proteobacteria phyla through conversion of L-carnitine or choline, found in red meats [52]. This is then converted to TMAO in the liver. High intake of red meat results in a higher amount of TMAO, which has been associated with the development of coronary artery disease, atherosclerosis, and stroke [44]. Similarly, inflammatory gut bacteria have also been correlated with elevated TMAO and coronary plaques [53, 54]. The hypothesis of the heart-gut axis is growing in acceptance and relates to the production of increased TMAO and endotoxins, and gut bacterial translocation in the progression of worsening heart failure and advancement of atherosclerotic disease $[28,55]$.

Butyrate is a short-chain fatty acid essential to the host immune homeostasis and a major energy source for intestinal epithelial cells [56]. The most abundant butyrate-producing organisms are Clostridium, Eubacterium, Fusobacterium, and Bifidobacterium [57-59]. The depletion of butyrate-producing organisms contributes to intestinal EBD and upregulated inflammatory responses in the body. Higher F/B ratio and elevations in Enterobacteriaceae have been associated with depleted quantities of butyrate-producing organisms, increased systemic inflammation, and stroke [60]. A diet high in fiber promotes butyrate-producing organism growth and has been shown to stabilize plaque size in atherosclerosis [59]. Heart failure and coronary artery disease have also been associated with a reduction in butyrate-producing organisms [59, 61].

Organisms of the oral and intestinal microbiome that are engaged in nitrogen respiration are an important source of nitric oxide (NO). Reactive oxygen and nitrogen species reduce nitrates and nitrites to deplete $\mathrm{NO}$ precursors and lead to impairment of NO-dependent vascular function $[62,63]$. If there is depletion of nitric oxide, due to either reduced production or increased consumption, this could contribute to pulmonary hypertension (PHTN); recent evidence is beginning to show a link between the microbiota and PHTN [64].

\section{The Microbiome and congenital Heart Disease}

Congenital heart disease, while separate from the above cardiovascular diseases, shares the risk for heart failure, hyper-thrombotic states, reduced splanchnic perfusion, and PHTN [65, 66]. Unique to CHD are complex lesions, namely single ventricle physiology, which will require a patient to undergo multiple surgeries using cardiopulmonary bypass (CPB) and live with hypoxemia for extended periods. Intestinal hypoxemia promotes 
inflammation, thereby inducing changes to the intestinal microbiome. Similar changes are observed during reduced blood flow $[67,68]$. Building from what is known in the adult population, similar processes and cardiovascular abnormalities can give insight into the microbiome's role in CHD and point to future study.

Intestinal epithelial barrier dysfunction has been associated with CPB [69]. This involves hypoxic, ischemic, or inflammatory injury to intestinal cells [70-73]. An intact intestinal barrier is a potent defense against enteric pathogens as well as inflammatory cytokines and toxins secreted from bacteria in the gut. Many patients with CHD experience impaired gut perfusion, either from poor cardiac output, hypoxemia-related vasoconstriction, or both. Intestinal dysbiosis may play a role in worsening EBD by enabling growth of LPS-producing bacteria, such as Escherichia coli and Enterobacteriaceae, leading to systemic inflammation [72]. Reduced gut perfusion and increased growth of Enterobacteriaceae are risk factors in the development of necrotizing enterocolitis [7, 74]. While necrotizing enterocolitis is a relatively uncommon pathology, it poses a risk to these patients and requires exposure to antibiotics, which can further disrupt the normal gut flora[74]. One mechanism for development of intestinal EBD includes inflammation following CPB leading to worsened dysbiosis and activation of cytokines and toxins from pro-inflammatory bacteria $[69,75,76]$. Roughly $25 \%$ of patients with CHD will require cardiac surgery with $\mathrm{CPB}$ [77]. We know existing dysbiosis is exacerbated following CPB [34]. Ischemia-induced EBD and dysbiosis, secondary to low cardiac output syndrome in the post-operative period, can also occur [78]. This may result in reduced populations of butyrate-producing organisms [79]. Butyrate is responsible for maintaining the intestinal barrier through modulation of G-coupled proteins and transcription factors, namely the nuclear transcription factor- $\mathrm{kB}(\mathrm{NF}-\mathrm{k} \mathrm{B})$ pathway, to upregulate regulatory T-cells. Loss of butyrate producing organisms can lead to EBD resulting in passive leak of lipopolysaccharides and other microbial toxins, which then bind to toll-like receptors on $\mathrm{T}$ cells activating the innate immune response [59, 61, 80, 81]. A third mechanism involves elevated TMAO production leading to EBD and activated inflammatory processes causing further cardiac injury [81-83]. TMAO is known to be associated with barrier dysfunction and activation of inflammatory cytokines [39, 84].

Hypoxemia and inflammatory insults are also known activators of hypoxia-inducible factor 1 (HIF-1) and NF$\kappa B$ [72]. HIF-1 is a transcription factor known as the master regulator of cellular and developmental responses to hypoxia [85]. NF- $\mathrm{kB}$ is a family of transcription factors involved in activation of cytokines and other inflammatory processes [86]. Organisms within
Proteobacteria and Firmicutes can upregulate the NF-кB pathway through binding of damage-associated and pathogen-associated molecular patterns found on epithelial cells, leading to vascular inflammation and cytokine activation [87-91]. During hypoxemic conditions, Enterobacteriaceae have been shown to increase both NF$\kappa \mathrm{B}$ and HIF- $1 \alpha$ signaling through interferon gamma activation, leading to de-regulation of intestinal epithelial tight junction proteins and activation of inflammatory cytokines [92-96]. Suppression of HIF-1 $\alpha$ improves intestinal EBD [72, 95]. HIF-1 $\alpha$ has been shown to be upregulated in both cyanotic and acyanotic cardiac lesions [97]. HIF- $1 \alpha$ and NF- $\mathrm{KB}$ regulate each other through both inflammatory and hypoxic activation [98100]. In patients with CHD, there can be inflammation from reduced splanchnic blood flow, hypoxemia, or a combination of both. Figure 2 illustrates how these mechanisms are involved in the development of systemic inflammation through intestinal dysbiosis and EBD under hypoxemic and ischemic conditions.

Bacteria and diet may also be involved in promoting PHTN. CHD is a high risk population for PHTN, occurring at rates of $10-30 \%$ [101]. Pro-inflammatory bacteria reduce circulating $\mathrm{NO}$ and NO precursors [102]. Disproportionate vasoconstriction and endothelial remodeling drives development of PHTN, and a reduction in bioavailability of NO signaling is a hallmark of PHTN [103]. Risks for developing this in CHD include excessive blood flow to the pulmonary system and a persistent hypoxemic state [104, 105]. Adult studies have shown the oral and intestinal flora correlate with increased risk of PHTN [64]. Certain organisms, such as Firmicutes and Actinobacteria, result in increased plasma arginase and ornithine transcarbamylase, thereby reducing the amount of circulating L-arginine, the major precursor of NO [103]. These same organisms are also involved in production of TMAO [64]. Proteobacteria induce further denitrification resulting in reduction beyond $\mathrm{NO}$ to $\mathrm{N}_{2} \mathrm{O}$ and $\mathrm{NH}_{3}$ [106]. Metabolism of nitrate is an important pathway for production of NO, and dysbiosis with elevated Enterobacteriaceae can consume NO reducing the availability of this potent vasodilator $[106,107]$.

Non-traditional signaling pathways involving microRNA (miRNA) also influence CHD and the microbiome $[108,109]$. There are miRNA's associated with intestinal dysbiosis, barrier dysfunction, and CHD. These small, evolutionally conserved, non-coding RNA regulate gene expression [110]. Specific miRNA have been identified to be involved in cardiac embryologic development, and their dysregulation can lead to structural abnormalities [111-117]. Upregulated miR-146 may reduce hypoxiainduced cardiomyocyte apoptosis through inhibition of the NF- $\kappa B$ pathway [118]. Studies have also found miRNA produced by intestinal epithelial cells regulate 


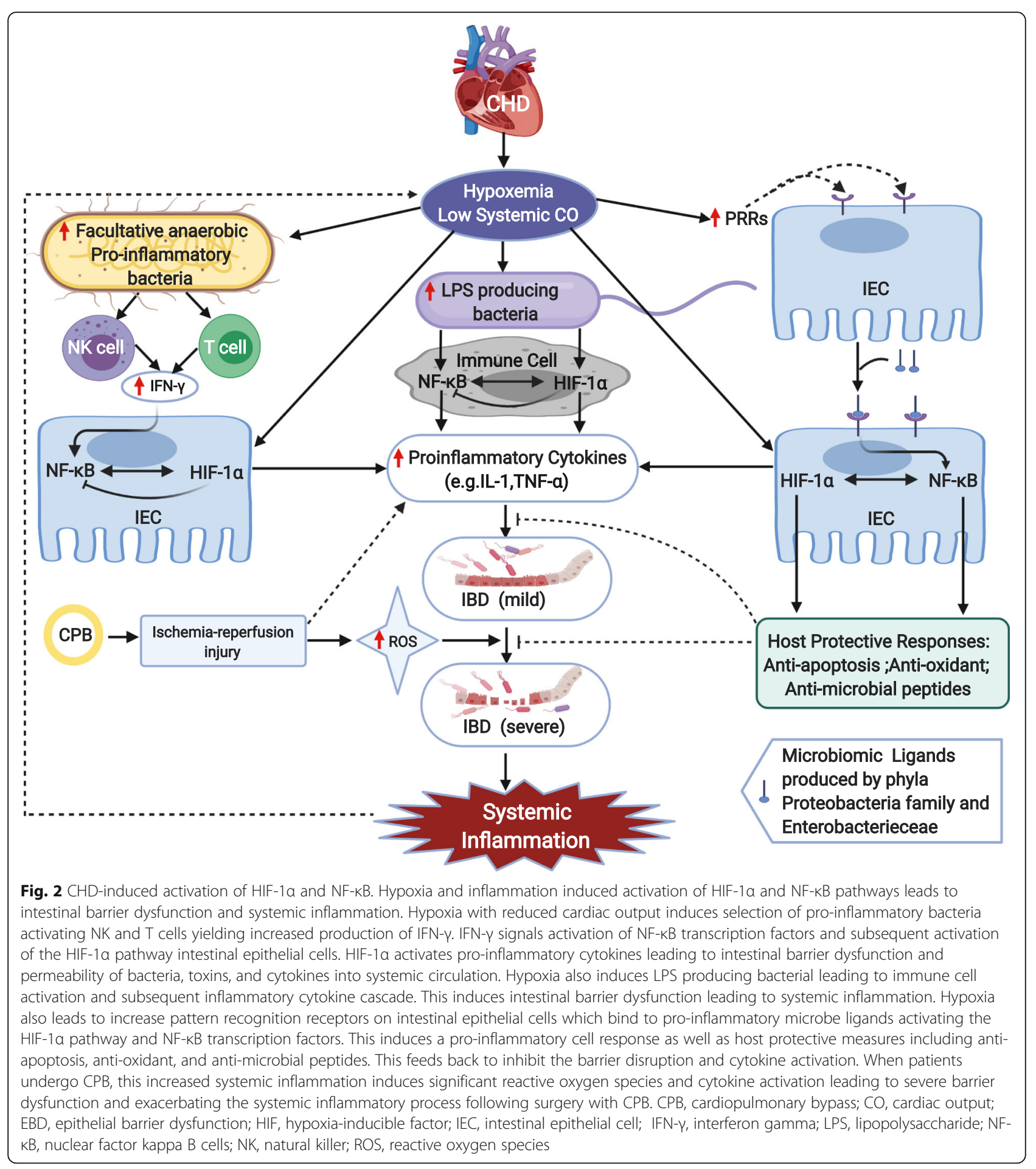

the expression of gut microbes [119-121]. MiR-515-5p and miR1226-5p promote growth of Fusobacterium nucleatum and Escherichia coli, respectively [119]. Both miR-191 and miR-212 are involved in intestinal EBD through disruption of zonula occludens-1, a protein involved in cell tight junctions [122, 123]. While miRNA have been identified in both the microbiome and CHD, no study has demonstrated miRNA with coordinated roles in both CHD and the microbiome. The overlap of miRNA in the microbiome, CHD, and EBD, as well as the complex role of miRNA in regulation of cardiac disease, is illustrated in Fig. 3. 


\section{Current gaps in knowledge}

Microbiome colonization and diversity is well documented in adult populations of cardiovascular disease, and we continue to learn more about its importance. Very little data exists evaluating the microbiome in CHD. Details related to microbial disparity in varying cardiac lesions, such as cyanotic and acyanotic lesions, and how these change following surgical repair and palliation will be important area of investigation. Dysbiosis has been identified in patients with CHD [32-34], but the effects of probiotics or synbiotics has only been briefly evaluated $[35,36]$. This area of research holds potential to identify therapeutic interventions aimed to improve morbidity and mortality in this patient population. Dilli and colleagues [35] demonstrated reduced sepsis, hospital length of stay, and mortality following the use of probiotics in patients with CHD. Probiotics have also been shown to reduce the degree of mucosal

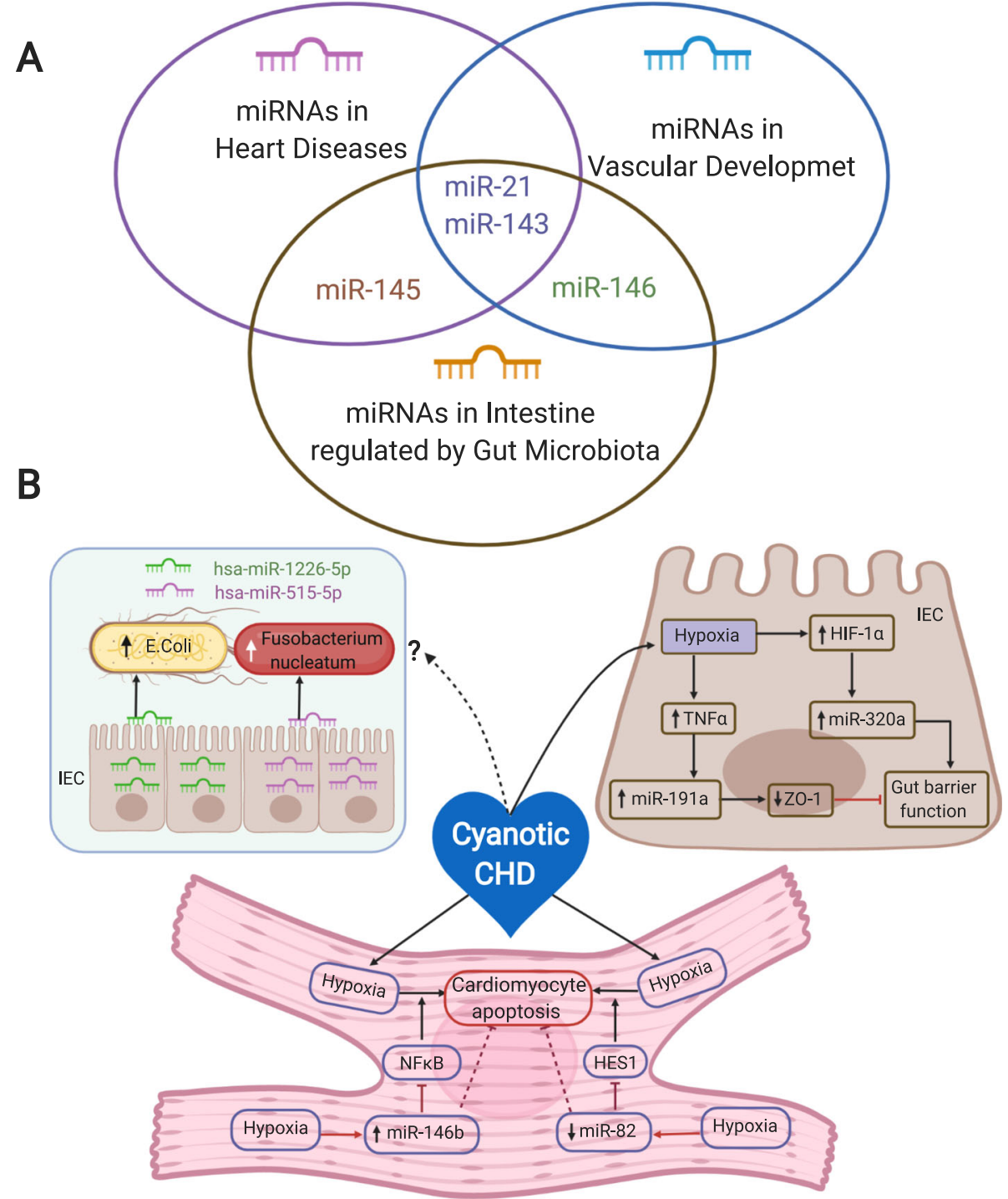

Fig. 3 Overlap of miRNA in the microbiome, CHD, and EBD. Panel a depicts the shared miRNA between cardiac disease and vascular development and the microbiome. These would be targets for study to evaluate links and similar mechanisms of action. Panel $\mathbf{b}$ demonstrates potential regulatory mechanisms of miRNA on cardiac and vascular function, intestinal epithelium, and the microbiome in CHD. MiR-1226-5p regulates growth of Fusobacterium nucleatum and miR-515-5p regulates growth of Escherichia coli. Hypoxia from CHD leads to activated HIF-1a, which in turn activates miR-320a. Hypoxia also leads to increased TNF-a which increases miRNA 191a and subsequent disruption of zonula occludens-1, an integral epithelial tight junction protein. Disruption of tight junction proteins leads to intestinal EBD. Hypoxia acts in the heart through NF-KB activated by increased miR-146b, which results in cardiomyocyte apoptosis. Protective features include over activation of miR-146b and reduced miR-82, which protect cardiomyocytes from apoptosis and cell death. MiRNA, micro ribonucleic acid; CHD, congenital heart disease; EBD, epithelial barrier dysfunction; HIF, hypoxia inducing factor; NF-KB, nuclear activating factor kappa B; IEC, intestinal epithelial cell 
inflammation following CPB in an animal study [124]. Rectified dysbiosis may also facilitate better regulation of maladaptive vasoconstriction to reduce the PHTN seen in these patients.

Further investigation is needed to evaluate signaling mechanisms for EBD, and to evaluate the relationship to stress, altered blood flow, and hypoxia leading to increased systemic inflammation. It is also unknown how small molecules, such as butyrate, TMAO, and nitrates influence inflammatory pathways, such as HIF-1 $\alpha$ and NF- $\mathrm{kB}$. Additionally, how these signaling pathways are upregulated in settings of cyanotic heart lesions is an important area which will improve our understanding of how systemic inflammation is generated. As EBD offers a mechanism for toxins and inflammatory metabolites to enter systemic circulation [125-127], methods to prevent or resolve the barrier dysfunction both pre- and post-operatively may also hold promise as therapeutic interventions.

Metagenomic and metabolomic are other tools available to help elucidate mechanisms of dysbiosis and enzyme activation in patients with $\mathrm{CHD}$, which will potentially discern linkages between altered gut flora and systemic inflammation. Biomarkers and proinflammatory mediators associated with pronounced systemic inflammation may yield targets by which to identify patients with higher risks of low cardiac output syndrome following CPB. This inflammatory cascade may be suppressed with improvements in the microbiota. There may also be a role to activate specific miRNA to promote bacterial growth and regulate inflammatory pathways. Studies to identify miRNA that

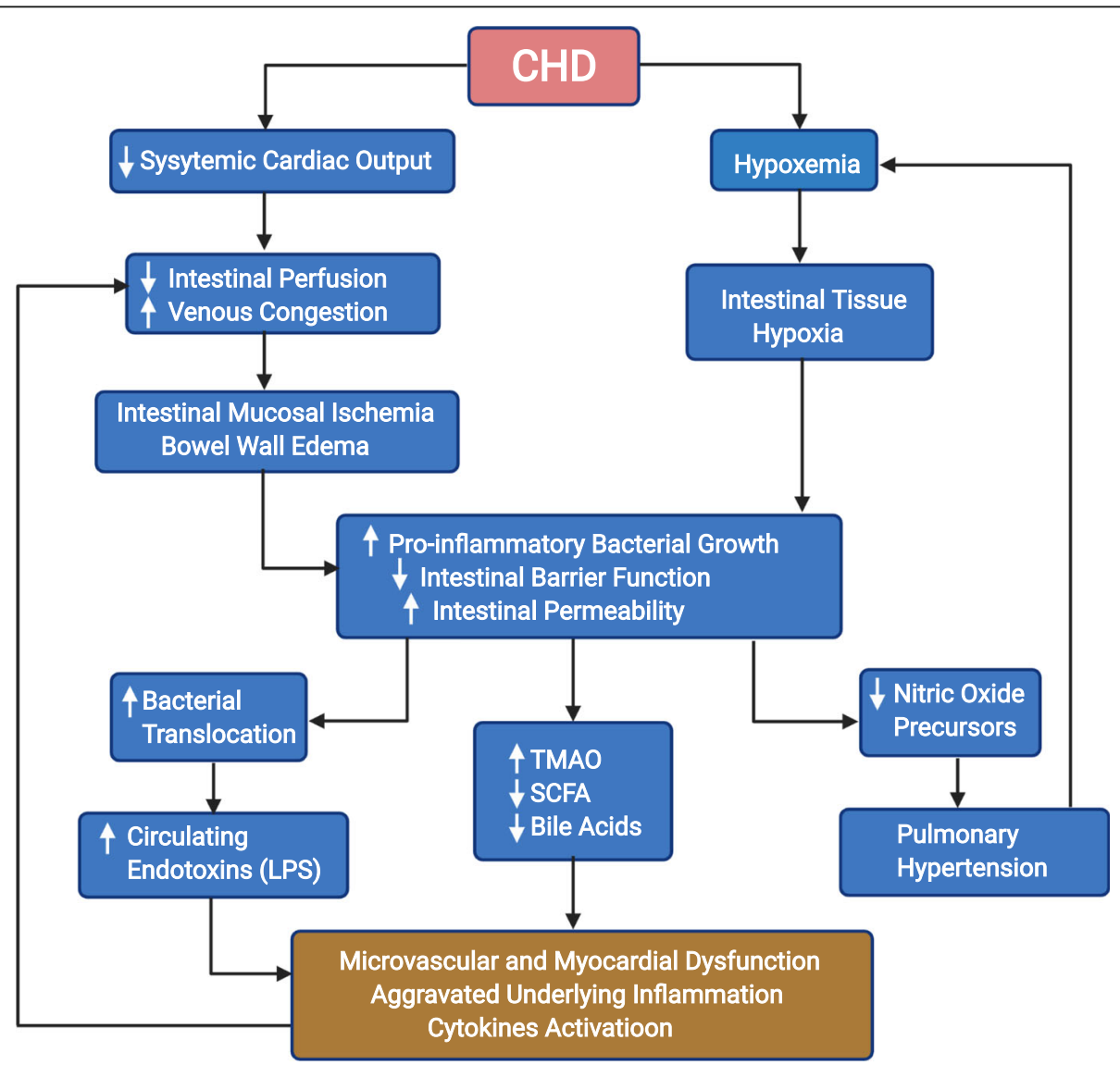

Fig. 4 Hypothesis of the heart-gut axis in congenital heart disease. Flow chart describing the pathogenesis of dysbiosis and the resulting systemic inflammation and end organ changes exacerbating the disease state in CHD. CHD causes reduced cardiac output. This reduced cardiac output leads to less intestinal perfusion and an increase in venous congestion as blood is not being pumped through the system as well. The resulting mucosal inflammation and edema yields an increase in pro-inflammatory growth, a reduction in intestinal barrier function, and increase intestinal permeability often described as a "leaky gut". This increased permeability leads to an increase in bacterial translocation and further leads to an increase in circulating toxins, such as LPS. The increased pro-inflammatory bacterial growth results in an increase in TMAO production through the liver, and a reduction in SCFA and secondary bile acids which are important for barrier regulation and cardiac health. The proinflammatory bacterial also engage in nitrogen respiratory which results in fewer nitric oxide precursors and can exacerbate the maladaptive response of pulmonary hypertension from an increase in pulmonary blood flow. The feedback loops exacerbate the pathology. CHD, congenital heart disease; LPS, lipopolysaccharide; SCFA, short chain fatty acid; TMAO, trimethylamine $\mathrm{N}$-oxide 
are involved in both CHD, the microbiome, and EBD would also improve our understanding of these processes as well as offer potential therapeutic targets to reduce inflammation in these patients. While studies regarding the influence of the microbiome and outcomes in CHD are lacking, there is importance evaluating interventions and expanding our understanding of these relationships. A flow chart indicating mechanisms and feedback promoting ongoing inflammation is provided in Fig. 4.

\section{Conclusions}

Intestinal dysbiosis is an important area of focus in patients with congenital heart disease and involves links with intestinal barrier dysfunction and systemic inflammation. Larger studies evaluating the microbiome in this patient population are needed to heighten the understanding of how changes in the microbiota affect CHD outcomes. This understanding will assist in development of animal models evaluating interventions on the microbiome and the degree of systemic inflammation following $\mathrm{CPB}$. Potential interventions include probiotics and synbiotics as well as the possibility of pre-operative fecal microbiome transplant to improve the gut proportion of healthy, gut-protective bacteria. Intestinal dysbiosis present in patients with CHD may be a contributing factor to the intestinal EBD following cardiac repair and $\mathrm{CPB}$. Improving the intestinal microbiome to reduce pro-inflammatory bacteria and increase butyrate producing organisms, reduce nitrate respiration, and modulate pathways such as HIF, NF- $\mathrm{kB}$, and miRNA are important aims for future investigation. These may play a key role in increasing our understanding of signaling mechanisms and identify therapeutic targets. There remains much to be understood about the microbiome's influence on homeostasis in CHD so that additional improvements in health, surgical outcomes, and the quality of life may be accomplished.

\section{Abbreviations \\ CHD: Congenital heart disease ; CPB: Cardiopulmonary bypass; EBD: Epithelial barrier dysfunction; F/B: Firmicutes/Bacteriodetes ratio; TMA: Trimethylamine; TMAO: Trimethylamine N-oxide; NO: Nitric oxide; LPS: Lipopolysaccharide; HIF: Hypoxia-inducible factor; NF-kB: Nuclear factor kappa B; mRNA: Micro-RNA}

\section{Acknowledgements}

Dr. Lindsey is a Stokes-Shackleford Professor at UNMC. The content is solely the responsibility of the authors and does not necessarily represent the official views of any of the funding agencies. All authors have reviewed and approved the article.

\section{Authors' contributions}

Dan Feng - Design, drafting and critical review/revision of the manuscript. Jason T. Christensen - Design, drafting and critical review/ revision of the manuscript. Anji T. Yetman - Drafting, and critical review/ revision of the manuscript. Merry L. Lindsey - Drafting and critical review/ revision of the manuscript. Amar B. Singh -Drafting and critical review/ revision of the manuscript. Jeffrey D. Salomon - Design, drafting and critical revision/review of the manuscript. The author(s) read and approved the final manuscript.

\section{Funding}

We acknowledge funding from the Child Health Research Institute Scholars Grant, UNMC, under award number 37-7215-6001-350. We also acknowledge funding from the National Institutes of Health under Award Numbers HL129823, HL137319, HL123471, and DK124095, and from the Biomedical Laboratory Research and Development Service of the Veterans Affairs Office of Research and Development under Award Numbers 5101BX000505 and BX002761B. The content is solely the responsibility of the authors and does not necessarily represent the official views of any of the funding agencies. All authors have reviewed and approved the article.

Availability of data and materials

Not Applicable.

\section{Declarations}

Ethics approval and Consent to participate

Not Applicable.

\section{Consent for publication}

Not Applicable.

\section{Competing interests}

The authors declare they have no competing interests.

\section{Author details}

${ }^{1}$ Department of Pediatrics, University of Nebraska Medical Center, Omaha, USA. ${ }^{2}$ Department of Internal Medicine, University of Nebraska Medical Center, Omaha, USA. ${ }^{3}$ Department of Cellular and Integrative Physiology, Center for Heart and Vascular Research, University of Nebraska Medical Center, Omaha, USA. ${ }^{4}$ Research Service, Nebraska-Western lowa Health Care System, Omaha, Nebraska, USA. ${ }^{5}$ Department of Molecular Biology and Biochemistry, University of Nebraska Medical Center, Omaha, USA. ${ }^{6}$ Child Health Research Institute, University of Nebraska Medical Center, Omaha, USA.

Received: 23 November 2020 Accepted: 12 March 2021

Published online: 14 April 2021

\section{References}

1. Petrini J, et al. Contribution of birth defects to infant mortality in the United States. Teratology. 2002;66 Suppl(1):3-6. doi:https://doi.org/10.1 002/tera.90002.

2. Gilboa S, Nembhard SJ, Fixler WN, Correa DE. A. Mortality Resulting From Congenital Heart Disease Among Children and Adults in the United States, 1999 to 2006. Circulation. 2010;122:2254-63.

3. Best KE, Rankin J. Long-Term Survival of Individuals Born With Congenital Heart Disease: A Systematic Review and Meta-Analysis. J Am Heart Assoc 5, doi:https://doi.org/10.1161/JAHA.115.002846 (2016).

4. Lopes S, et al. Mortality for Critical Congenital Heart Diseases and Associated Risk Factors in Newborns. A Cohort Study. Arq Bras Cardiol. 2018; 111:666-73. doi:https://doi.org/10.5935/abc.20180175.

5. McCracken $\mathrm{C}$, et al. Mortality Following Pediatric Congenital Heart Surgery: An Analysis of the Causes of Death Derived From the National Death Index. J Am Heart Assoc. 2018;7:e010624. doi:https://doi.org/10.11 61/JAHA.118.010624.

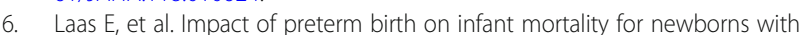
congenital heart defects: The EPICARD population-based cohort study. BMC Pediatr. 2017;17:124. doi:https://doi.org/10.1186/s12887-017-0875-z.

7. McElhinney DB, et al. Necrotizing enterocolitis in neonates with congenital heart disease: risk factors and outcomes. Pediatrics. 2000;106:1080-7. doi: https://doi.org/10.1542/peds.106.5.1080.

8. Chew MS, Ravn B-CV, Brandslund HB, Ditlevsen I. E, et al. Effect of modified ultrafiltration on the inflammatory response in paediatric open-heart surgery: a prospective, randomized study. Perfusion 17(5):327-33 (2002). 
9. Gaynor J. The effect of modified ultrafiltration on the postoperative course in patients with congenital heart disease. Seminars in Thoracic Cardiovascular Surgery: Pediatric Cardiac Surgery Annual. 2003;6:128-39.

10. Hill A, et al. Current Evidence about Nutrition Support in Cardiac Surgery Patients-What Do We Know? Nutrients10, doi:https://doi.org/10.3390/nu1 0050597 (2018).

11. Lomivorotov W, et al. Evaluation of nutritional screening tools for patients scheduled for cardiac surgery. Nutrition. 2013;29:436-42. doi:https://doi. org/10.1016/j.nut.2012.08.006.

12. Stoppe $C$, et al. Role of nutrition support in adult cardiac surgery: a consensus statement from an International Multidisciplinary Expert Group on Nutrition in Cardiac Surgery. Crit Care. 2017;21:131. doi:https://doi.org/1 0.1186/s13054-017-1690-5.

13. Agarwal HS, Wolfram KB, Saville BR, Donahue BS, Bichell DP. Postoperative complications and association with outcomes in pediatric cardiac surgery. J Thorac Cardiovasc Surg. 2014;148(e601):609-16. doi:https://doi.org/10.1016/j. jtcvs.2013.10.031

14. Mubayed L, Al-Kindi S. Recent Trends in Infant Mortality due to Congenital Heart Disease in the United States. Pediatrics. 2019;144:344-4. doi:https:// doi.org/10.1542/peds.144.2_MeetingAbstract.344.

15. Kamada N, Chen GY, Inohara N, Nunez G. Control of pathogens and pathobionts by the gut microbiota. Nat Immunol. 2013;14:685-90. doi: https://doi.org/10.1038/ni.2608.

16. Bull MJ, Plummer NT. Part 1: The Human Gut Microbiome in Health and Disease. Integr Med (Encinitas). 2014;13:17-22.

17. Round $\lrcorner$, Mazmanian SK. The gut microbiota shapes intestinal immune responses during health and disease. Nat Rev Immunol. 2009;9:313-23. doi: https://doi.org/10.1038/nri2515.

18. Wang H, Wei C-X, Min L, Zhu L-Y. Good or bad: gut bacteria in human health and diseases. Biotechnology Biotechnological Equipment. 2018;32: 1075-80. doi:https://doi.org/10.1080/13102818.2018.1481350.

19. Alverdy JC, Krezalek MA. Collapse of the Microbiome, Emergence of the Pathobiome, and the Immunopathology of Sepsis. Crit Care Med. 2017:45: 337-47. doi:https://doi.org/10.1097/CCM.0000000000002172.

20. Cabrera-Perez J, Badovinac VP, Griffith TS. Enteric immunity, the gut microbiome, and sepsis: Rethinking the germ theory of disease. Exp Biol Med (Maywood). 2017;242:127-39. doi:https://doi.org/10.1177/1535370216669610.

21. Shimizu K, et al. Altered gut flora are associated with septic complications and death in critically ill patients with systemic inflammatory response syndrome. Dig Dis Sci. 2011;56:1171-7. doi:https://doi.org/10.1007/s10620-01 0-1418-8.

22. Aardema H, et al. Marked Changes in Gut Microbiota in Cardio-Surgical Intensive Care Patients: A Longitudinal Cohort Study. Front Cell Infect Microbiol. 2019;9:467. doi:https://doi.org/10.3389/fcimb.2019.00467.

23. Zheng S, et al. Clinical Parameters and Gut Microbiome Changes Before and After Surgery in Thoracic Aortic Dissection in Patients with Gastrointestinal Complications. Sci Rep. 2017;7:15228. doi:https://doi.org/10.1038/s41598-01 7-15079-0.

24. Ding W, et al. Clinical Multi-Omics Study on the Gut Microbiota in Critically III Patients After Cardiovascular Surgery Combined With Cardiopulmonary Bypass With or Without Sepsis (MUL-GM-CSCPB Study): A Prospective Study Protocol. Front Med (Lausanne). 2020;7:269. doi:https://doi.org/10.3389/ fmed.2020.00269.

25. Cenit MC, Sanz Y, Codoner-Franch P. Influence of gut microbiota on neuropsychiatric disorders. World J Gastroenterol. 2017;23:5486-98. doi: https://doi.org/10.3748/wjg.v23.i30.5486.

26. De Luca F, Shoenfeld $Y$. The microbiome in autoimmune diseases. Clin Exp Immunol. 2019;195:74-85. doi:https://doi.org/10.1111/cei.13158.

27. Ley RE, et al. Obesity alters gut microbial ecology. Proc Natl Acad Sci U S A. 2005;102:11070-5. doi:https://doi.org/10.1073/pnas.0504978102.

28. Harikrishnan S. Diet, the Gut Microbiome and Heart Failure. Card Fail Rev. 2019;5:119-22. doi:https://doi.org/10.15420/cfr.2018.39.2.

29. Tang WH, Kitai T, Hazen SL. Gut Microbiota in Cardiovascular Health and Disease. Circ Res. 2017;120:1183-96. doi:https://doi.org/10.1161/CIRCRESA HA.117.309715.

30. Tang WHW, Backhed F, Landmesser U, Hazen SL. Intestinal Microbiota in Cardiovascular Health and Disease: JACC State-of-the-Art Review. J Am Coll Cardiol. 2019;73:2089-105. doi:https://doi.org/10.1016/j.jacc.2019.03.024.

31. Pathan $\mathrm{N}$, et al. Intestinal injury and endotoxemia in children undergoing surgery for congenital heart disease. Am J Respir Crit Care Med. 2011;184: 1261-9. doi:https://doi.org/10.1164/rccm.201104-07150C.
32. Patterson S, et al GASTROINTESTINAL MICROBIOTA DIVERSITY AND CLINICAL OUTCOMES IN CONGENITAL HEART DISEASE. Crit Care Med 48, doi:https:// doi.org/10.1097/01.ccm.0000619040.61766.26 (2020).

33. Salomon J, Price A, Manithody C, Jain A, EFFECT OF CARDIOPULMONARY, BYPASS ON THE PEDIATRIC INTESTINAL MICROBIOME. Crit Care Med 47, doi: https://doi.org/10.1097/01.ccm.0000551206.38953.82 (2019).

34. Salomon J, Price A, Manithody C, Jain A. Clonal Proliferation of Proinflammatory Gut Bacteria and Alteration in Gut Permiability are Associated with Cardiopulmonary Bypass Surgery in Children. Journal of Pediatric Gastroenterology and Nutrition 69 (2019).

35. Dilli $\mathrm{D}$, et al. Treatment outcomes of infants with cyanotic congenital heart disease treated with synbiotics. Pediatrics. 2013;132:e932-8. doi:https://doi. org/10.1542/peds.2013-1262.

36. Ellis $\mathrm{CL}$, et al. Probiotic administration in congenital heart disease: a pilot study. J Perinatol. 2013;33:691-7. doi:https://doi.org/10.1038/jp.2013.41.

37. Thursby E, Juge N. Introduction to the human gut microbiota. Biochem J. 2017:474:1823-36. doi:https://doi.org/10.1042/BCJ20160510.

38. Qin J, et al. A human gut microbial gene catalogue established by metagenomic sequencing. Nature. 2010;464:59-65. https://doi.org/10.1038/nature08821.

39. Rizzatti G, Lopetuso LR, Gibiino G, Binda C, Gasbarrini A, Proteobacteria: A Common Factor in Human Diseases. Biomed Res Int 2017, 9351507, doi: https://doi.org/10.1155/2017/9351507 (2017).

40. Mariat $\mathrm{D}$, et al. The Firmicutes/Bacteroidetes ratio of the human microbiota changes with age. BMC Microbiol. 2009;9:123. doi:https://doi.org/10.1186/14 71-2180-9-123.

41. Razavi AC, Potts KS, Kelly TN, Bazzano LA. Sex, gut microbiome, and cardiovascular disease risk. Biol Sex Differ. 2019;10:29. doi:https://doi.org/1 0.1186/s13293-019-0240-z.

42. Ridlon JM, Kang DJ, Hylemon PB, Bajaj JS. Bile acids and the gut microbiome. Curr Opin Gastroenterol. 2014;30:332-8. doi:https://doi.org/10.1 097/MOG.0000000000000057.

43. Dieterich $W$, Schink $M$, Zopf Y. Microbiota in the Gastrointestinal Tract. Med Sci (Basel) 6, doi:https://doi.org/10.3390/medsci6040116 (2018).

44. Brown JM, Hazen SL. Microbial modulation of cardiovascular disease. Nat Rev Microbiol. 2018;16:171-81. doi:https://doi.org/10.1038/nrmicro.2017.149.

45. Amar J, et al. Blood microbiota dysbiosis is associated with the onset of cardiovascular events in a large general population: the D.E.S.I.R. study. PLOS One. 2013:8:e54461. doi:https://doi.org/10.1371/journal.pone.0054461.

46. Brown JM, Hazen SL. The gut microbial endocrine organ: bacterially derived signals driving cardiometabolic diseases. Annu Rev Med. 2015;66:343-59. doi:https://doi.org/10.1146/annurev-med-060513-093205.

47. Taniyama Y, Griendling KK. Reactive oxygen species in the vasculature: molecular and cellular mechanisms. Hypertension. 2003;42:1075-81. doi: https://doi.org/10.1161/01.HYP.0000100443.09293.4F.

48. Munzel T, et al. Impact of Oxidative Stress on the Heart and Vasculature: Part 2 of a 3-Part Series. J Am Coll Cardiol. 2017;70:212-29. doi:https://doi. org/10.1016/j.jacc.2017.05.035.

49. Jie $Z$, et al. The gut microbiome in atherosclerotic cardiovascular disease. Nat Commun. 2017:8:845. doi:https://doi.org/10.1038/s41467-017-00900-1.

50. Forkosh $E$, llan $Y$. The heart-gut axis: new target for atherosclerosis and congestive heart failure therapy. Open Heart. 2019;6:e000993. doi:https://doi. org/10.1136/openhrt-2018-000993.

51. Kitai T, Kirsop J, Tang WH. Exploring the Microbiome in Heart Failure. Curr Heart Fail Rep. 2016;13:103-9. doi:https://doi.org/10.1007/s11897-016-0285-9.

52. Rath S, Rud T, Pieper DH, Vital M. Potential TMA-Producing Bacteria Are Ubiquitously Found in Mammalia. Front Microbiol. 2019;10:2966. doi:https:// doi.org/10.3389/fmicb.2019.02966.

53. Cho CE, et al. Trimethylamine-N-oxide (TMAO) response to animal source foods varies among healthy young men and is influenced by their gut microbiota composition: A randomized controlled trial. Mol Nutr Food Res 61, doi:https://doi.org/10.1002/mnfr.201600324 (2017).

54. Fu Q, et al. Coronary Plaque Characterization Assessed by Optical Coherence Tomography and Plasma Trimethylamine-N-oxide Levels in Patients With Coronary Artery Disease. Am J Cardiol. 2016;118:1311-5. doi: https://doi.org/10.1016/j.amjcard.2016.07.071.

55. Tang WHW, Li DY, Hazen SL. Dietary metabolism, the gut microbiome, and heart failure. Nat Rev Cardiol. 2019;16:137-54. doi:https://doi.org/10.1038/s41 569-018-0108-7.

56. Moens $F$, et al. A four-strain probiotic exerts positive immunomodulatory effects by enhancing colonic butyrate production in vitro. Int J Pharm. 2019; 555:1-10. doi:https://doi.org/10.1016/j.jpharm.2018.11.020. 
57. Riviere A, Selak M, Lantin D, Leroy F, De Vuyst L. Bifidobacteria and ButyrateProducing Colon Bacteria: Importance and Strategies for Their Stimulation in the Human Gut. Front Microbiol. 2016;7:979. doi:https://doi.org/10.3389/ fmicb.2016.00979.

58. Vital M, Karch A, Pieper DH. Colonic Butyrate-Producing Communities in Humans: an Overview Using Omics Data. mSystems 2, doi:https://doi.org/1 0.1128/mSystems.00130-17 (2017)

59. Xiao Y, Guo Z, Li Z, Ling H, Song C. Role and Mechanism of Action of Butyrate in Atherosclerotic diseases: A Review. J Appl Microbiol. 2020. doi: https://doi.org/10.1111/jam.14906.

60. Zeng $X$, et al. Higher Risk of Stroke Is Correlated With Increased Opportunistic Pathogen Load and Reduced Levels of Butyrate-Producing Bacteria in the Gut. Front Cell Infect Microbiol 9, 4, doi:https://doi.org/10.33 89/fcimb.2019.00004 (2019).

61. Troseid M, Andersen GO, Broch K, Hov JR. The gut microbiome in coronary artery disease and heart failure: Current knowledge and future directions. EBioMedicine. 2020;52:102649. doi:https://doi.org/10.1016/j.ebiom.2020.102649.

62. Scales BS, Dickson RP, Huffnagle GB. A tale of two sites: how inflammation can reshape the microbiomes of the gut and lungs. J Leukoc Biol. 2016;100: 943-50. doi:https://doi.org/10.1189/jlb.3MR0316-106R.

63. Tiso $M$, Schechter AN. Nitrate reduction to nitrite, nitric oxide and ammonia by gut bacteria under physiological conditions. PLoS One. 2015;10: e0119712. doi:https://doi.org/10.1371/journal.pone.0119712.

64. Kim S, et al. Altered Gut Microbiome Profile in Patients With Pulmonary Arterial Hypertension. Hypertension. 2020;75:1063-71. doi:https://doi.org/1 0.1161/HYPERTENSIONAHA.119.14294.

65. Hinton RB, Ware SM. Heart Failure in Pediatric Patients With Congenital Heart Disease. Circ Res. 2017;120:978-94. doi:https://doi.org/10.1161/ CIRCRESAHA.116.308996

66. Wang T, et al. Congenital Heart Disease and Risk of Cardiovascular Disease: A Meta-Analysis of Cohort Studies. J Am Heart Assoc. 2019;8:e012030. doi: https://doi.org/10.1161/JAHA.119.012030.

67. Moreno-Indias I, et al. Intermittent hypoxia alters gut microbiota diversity in a mouse model of sleep apnoea. Eur Respir J. 2015;45:1055-65. doi:https:// doi.org/10.1183/09031936.00184314.

68. Wang F, Li Q, Wang C, Tang C, Li J. Dynamic alteration of the colonic microbiota in intestinal ischemia-reperfusion injury. PLoS One. 2012;7: e42027. doi:https://doi.org/10.1371/journal.pone.0042027.

69. Typpo KV, et al. Clinical characteristics associated with postoperative intestinal epithelial barrier dysfunction in children with congenital heart disease. Pediatr Crit Care Med. 2015;16:37-44. doi:https://doi.org/10.1097/ PCC.0000000000000256.

70. Glover LE, Lee JS, Colgan SP. Oxygen metabolism and barrier regulation in the intestinal mucosa. J Clin Invest. 2016:126:3680-8. doi:https://doi.org/1 $0.1172 / \mathrm{JCl} 84429$

71. Lee TC, Huang YC, Lu YZ, Yeh YC, Yu LC. Hypoxia-induced intestinal barrier changes in balloon-assisted enteroscopy. J Physiol. 2018;596:3411-24. doi: https://doi.org/10.1113/JP275277.

72. Lei $\mathrm{Q}$, et al. Amelioration of hypoxia and LPS-induced intestinal epithelial barrier dysfunction by emodin through the suppression of the NF-kappaB and HIF-1alpha signaling pathways. Int J Mol Med. 2014;34:1629-39. doi: https://doi.org/10.3892/ijmm.2014.1965.

73. Zeitouni NE, Chotikatum S, von Kockritz-Blickwede M, Naim HY. The impact of hypoxia on intestinal epithelial cell functions: consequences for invasion by bacterial pathogens. Mol Cell Pediatr. 2016;3:14. doi:https://doi.org/10.11 86/s40348-016-0041-y.

74. Coggins SA, Wynn JL, Weitkamp JH. Infectious causes of necrotizing enterocolitis. Clin Perinatol 42, 133-154, ix, doi:https://doi.org/10.1016/j.clp.2 014.10.012 (2015).

75. Cremer J, et al. Systemic inflammatory response syndrome after cardiac operations. The Annals of Thoracic Surgery. 1996;61:1714-20. doi:https://doi. org/10.1016/0003-4975(96)00055-0.

76. Halter J, Fink SJ, Lutz G, Picone C, Maybury A, Fedors R, DiRocco N, Lee J, Nieman HM. G. Evidence of systemic cytokine release in patients undergoing cardiopulmonary bypass. The Journal of Extracorporeal Technology. 2005;37:272-7.

77. Oster ME, et al. Temporal trends in survival among infants with critical congenital heart defects. Pediatrics. 2013;131:e1502-8. doi:https://doi.org/1 0.1542/peds.2012-3435.

78. Lim SH, tyrate, Propionate. Short Chain Fatty Acids, Attenuate Myocardial Damages by Inhibition of Apoptosis in a Rat Model of Ischemia-reperfusion.
Journal of the Korean Society for Applied Biological Chemistry. 2010;53:5707. doi:https://doi.org/10.3839/jksabc.2010.088.

79. Luedde $\mathrm{M}$, et al. Heart failure is associated with depletion of core intestinal microbiota. ESC Heart Fail. 2017;4:282-90. doi:https://doi.org/10.1002/ ehf2.12155.

80. Bedford A, Gong J. Implications of butyrate and its derivatives for gut health and animal production. Anim Nutr. 2018:4:151-9. doi:https://doi.org/10.101 6/j.aninu.2017.08.010.

81. Zhang $L$, et al. Sodium butyrate attenuates angiotensin II-induced cardiac hypertrophy by inhibiting COX2/PGE2 pathway via a HDAC5/HDAC6dependent mechanism. J Cell Mol Med. 2019;23:8139-50. doi:https://doi. org/10.1111/jcmm.14684.

82. Jia Q, et al. Role and Effective Therapeutic Target of Gut Microbiota in Heart Failure. Cardiovasc Ther 2019, 5164298, doi:https://doi.org/10.1155/2019/51 64298 (2019).

83. Zhang L, et al. Sodium Butyrate Protects -Against High Fat Diet-Induced Cardiac Dysfunction and Metabolic Disorders in Type II Diabetic Mice. J Cell Biochem. 2017;118:2395-408. doi:https://doi.org/10.1002/jcb.25902.

84. Canyelles $\mathrm{M}$, et al. Trimethylamine N-Oxide: A Link among Diet, Gut Microbiota, Gene Regulation of Liver and Intestine Cholesterol Homeostasis and HDL Function. Int J Mol Sci 19, doi:https://doi.org/1 0.3390/ijms19103228 (2018).

85. Iyer NV, et al. Cellular and developmental control of $\mathrm{O} 2$ homeostasis by hypoxia-inducible factor 1 alpha. Genes Dev. 1998;12:149-62. doi:https://doi. org/10.1101/gad.12.2.149.

86. Gilmore TD. Introduction to NF-kappaB: players, pathways, perspectives. Oncogene. 2006:25:6680-4. doi:https://doi.org/10.1038/sj.onc.1209954.

87. Kaci G, et al. Inhibition of the NF-kappaB pathway in human intestinal epithelial cells by commensal Streptococcus salivarius. Appl Environ Microbiol. 2011;77:4681-4. doi:https://doi.org/10.1128/AEM.03021-10.

88. Segain JP, et al. Butyrate inhibits inflammatory responses through NFkappaB inhibition: implications for Crohn's disease. Gut. 2000;47:397-403. doi:https:// doi.org/10.1136/gut.47.3.397.

89. Seldin MM, et al. Trimethylamine N-Oxide Promotes Vascular Inflammation Through Signaling of Mitogen-Activated Protein Kinase and Nuclear FactorkappaB. J Am Heart Assoc 5, doi:https://doi.org/10.1161/JAHA.115.002767 (2016).

90. Tato CM, Hunter CA. Host-pathogen interactions: subversion and utilization of the NF-kappa B pathway during infection. Infect Immun. 2002;70:3311-7. doi:https://doi.org/10.1128/iai.70.7.3311-3317.2002.

91. Wullaert A, Bonnet MC, Pasparakis M. NF-kappaB in the regulation of epithelial homeostasis and inflammation. Cell Res. 2011;21:146-58. doi: https://doi.org/10.1038/cr.2010.175.

92. Hartmann $\mathrm{H}$, et al. Hypoxia-independent activation of HIF-1 by enterobacteriaceae and their siderophores. Gastroenterology. 2008;134:75667. doi:https://doi.org/10.1053/j.gastro.2007.12.008.

93. Pott J, Stockinger S, Type I. Interferon in the Gut: Tight Balance between Host Protection and Immunopathology. Front Immunol. 2017:8:258. doi: https://doi.org/10.3389/fimmu.2017.00258. and III.

94. Santos SAD, Andrade DRJ. HIF-1alpha and infectious diseases: a new frontier for the development of new therapies. Rev Inst Med Trop Sao Paulo. 2017; 59:e92. doi:https://doi.org/10.1590/S1678-9946201759092.

95. Weidemann A, Johnson RS. Biology of HIF-1alpha. Cell Death Differ. 2008;15: 621-7. doi:https://doi.org/10.1038/cdd.2008.12

96. Yang $\mathrm{S}$, et al. Interferon-gamma-induced intestinal epithelial barrier dysfunction by NF-kappaB/HIF-1alpha pathway. J Interferon Cytokine Res. 2014;34:195-203. doi:https://doi.org/10.1089/jir.2013.0044.

97. Qing M, et al. The hypoxia-inducible factor HIF-1 promotes intramyocardial expression of VEGF in infants with congenital cardiac defects. Basic Res Cardiol. 2007;102:224-32. doi:https://doi.org/10.1007/s00395-007-0639-2.

98. Bandarra D, Biddlestone J, Mudie S, Muller HA, Rocha S. HIF-1alpha restricts NF-kappaB-dependent gene expression to control innate immunity signals. Dis Model Mech. 2015;8:169-81. doi:https://doi.org/10.1242/dmm.017285.

99. D'lgnazio L, Rocha S. Hypoxia Induced NF-kappaB. Cells 5, doi:https://doi. org/10.3390/cells5010010 (2016).

100. Imtiyaz HZ, Simon MC. Hypoxia-inducible factors as essential regulators of inflammation. Curr Top Microbiol Immunol. 2010;345:105-20. doi:https://doi. org/10.1007/82_2010_74.

101. Frogoudaki AA, Gatzoulis MA. Pulmonary arterial hypertension in congenital heart disease. Continuing Cardiology Education. 2018:4:23-33. doi:https:// doi.org/10.1002/cce2.74. 
102. Bueno M, Wang J, Mora AL, Gladwin MT. Nitrite signaling in pulmonary hypertension: mechanisms of bioactivation, signaling, and therapeutics. Antioxid Redox Signal. 2013;18:1797-809. doi:https://doi.org/10.1089/ars.2 012.4833 .

103. Koch CD, et al. Enterosalivary nitrate metabolism and the microbiome: Intersection of microbial metabolism, nitric oxide and diet in cardiac and pulmonary vascular health. Free Radic Biol Med. 2017;105:48-67. doi:https:// doi.org/10.1016/j.freeradbiomed.2016.12.015.

104. Kulik TJ. Pulmonary blood flow and pulmonary hypertension: Is the pulmonary circulation flowophobic or flowophilic? Pulm Circ. 2012;2:327-39. doi:https://doi.org/10.4103/2045-8932.101644.

105. Pascall E, Tulloh RM. Pulmonary hypertension in congenital heart disease. Future Cardiol. 2018;14:343-53. doi:https://doi.org/10.2217/fca-2017-0065.

106. Doel JJ, Benjamin N, Hector MP, Rogers M, Allaker RP. Evaluation of bacterial nitrate reduction in the human oral cavity. Eur J Oral Sci. 2005;113:14-9. doi: https://doi.org/10.1111/j.1600-0722.2004.00184.x.

107. Sparacino-Watkins C, Stolz JF, Basu P. Nitrate and periplasmic nitrate reductases. Chem Soc Rev. 2014;43:676-706. doi:https://doi.org/10.1039/c3 cs60249d.

108. Bi K, Zhang X, Chen W, Diao H. MicroRNAs Regulate Intestinal Immunity and Gut Microbiota for Gastrointestinal Health: A Comprehensive Review. Genes (Basel) 11, doi:https://doi.org/10.3390/genes1 1091075 (2020).

109. Small EM, Olson EN. Pervasive roles of microRNAs in cardiovascular biology. Nature. 2011;469:336-42. doi:https://doi.org/10.1038/nature09783.

110. Bartel DP, MicroRNAs. Cell. 2004;116:281-97. doi:https://doi.org/10.1016/ s0092-8674(04)00045-5.

111. Li D, et al. Characterization of circulating microRNA expression in patients with a ventricular septal defect. PLoS One. 2014;9:e106318. doi:https://doi. org/10.1371/journal.pone.0106318.

112. Li J, et al. Roles of miR-1-1 and miR-181c in ventricular septal defects. Int J Cardiol. 2013;168:1441-6. doi:https://doi.org/10.1016/j.ijcard.2012.12.048.

113. O'Brien JE Jr, et al. Noncoding RNA expression in myocardium from infants with tetralogy of Fallot. Circ Cardiovasc Genet. 2012;5:279-86. doi:https:// doi.org/10.1161/CIRCGENETICS.111.961474

114. Smith T, Rajakaruna C, Caputo M, Emanueli C. MicroRNAs in congenital heart disease. Ann Transl Med. 2015;3:333. doi:https://doi.org/10.3978/j. issn.2305-5839.2015.12.25.

115. Vishal Nigam MD, Hans H, Sievers, Brian C, Jensen MD, Holger A, Sier PC, Simpson MD, Srivastava D, Salah MD. A. Mohamed. Altered Micrornas in Bicuspid Aortic Valve: A Comparison between Stenotic and Insufficient Valves. Journal of Heart Valve Disease 19 (2010).

116. Yanagawa B, et al. miRNA-141 is a novel regulator of BMP-2-mediated calcification in aortic stenosis. J Thorac Cardiovasc Surg. 2012;144:256-62. doi:https://doi.org/10.1016/j.jtcvs.2011.10.097.

117. Zhu $\mathrm{S}$, et al. Identification of maternal serum microRNAs as novel noninvasive biomarkers for prenatal detection of fetal congenital heart defects. Clin Chim Acta. 2013;424:66-72. doi:https://doi.org/10.1016/j. cca.2013.05.010

118. Li JW, et al. MicroRNA-146b inhibition augments hypoxia-induced cardiomyocyte apoptosis. Mol Med Rep. 2015;12:6903-10. doi:https://doi. org/10.3892/mmr.2015.4333.

119. Liu S, et al. The Host Shapes the Gut Microbiota via Fecal MicroRNA. Cell Host Microbe. 2016;19:32-43. doi:https://doi.org/10.1016/j.chom.201 5.12.005.

120. Liu S, Weiner HL. Control of the gut microbiome by fecal microRNA. Microb Cell. 2016;3:176-7. doi:https://doi.org/10.15698/mic2016.04.492.

121. Moloney GM, Viola MF, Hoban AE, Dinan TG, Cryan JF. Faecal microRNAs: indicators of imbalance at the host-microbe interface? Benef Microbes. 2018;9:175-83. doi:https://doi.org/10.3920/BM2017.0013.

122. Tang Y, et al. The Role of miR-212 and iNOS in Alcohol-Induced Intestinal Barrier Dysfunction and Steatohepatitis. Alcohol Clin Exp Res. 2015;39:163241. doi:https://doi.org/10.1111/acer.12813.

123. Wang L, Zhang R, Chen J, Wu Q, Kuang Z. Baicalin Protects against TNFalpha-Induced Injury by Down-Regulating miR-191a That Targets the Tight Junction Protein ZO-1 in IEC-6 Cells. Biol Pharm Bull. 2017:40:435-43. doi: https://doi.org/10.1248/bpb.b16-00789.

124. Sun YJ, et al. Probiotics can alleviate cardiopulmonary bypass-induced intestinal mucosa damage in rats. Dig Dis Sci. 2013;58:1528-36. doi:https:// doi.org/10.1007/s10620-012-2546-0.

125. Lobionda S, Sittipo P, Kwon HY, Lee YK. The Role of Gut Microbiota in Intestinal Inflammation with Respect to Diet and Extrinsic Stressors.
Microorganisms 7, doi:https://doi.org/10.3390/microorganisms7080271 (2019).

126. Mittal R, Coopersmith CM. Redefining the gut as the motor of critical illness. Trends Mol Med. 2014;20:214-23. doi:https://doi.org/10.1016/j. molmed.2013.08.004.

127. Perrone EE, et al. Mechanisms of methicillin-resistant Staphylococcus aureus pneumonia-induced intestinal epithelial apoptosis. Shock. 2012;38:68-75. doi:https://doi.org/10.1097/SHK.0b013e318259abdb.

\section{Publisher's Note}

Springer Nature remains neutral with regard to jurisdictional claims in published maps and institutional affiliations.
Ready to submit your research? Choose BMC and benefit from:

- fast, convenient online submission

- thorough peer review by experienced researchers in your field

- rapid publication on acceptance

- support for research data, including large and complex data types

- gold Open Access which fosters wider collaboration and increased citations

- maximum visibility for your research: over $100 \mathrm{M}$ website views per year

At BMC, research is always in progress.

Learn more biomedcentral.com/submissions 